\title{
Obesity and urinary incontinence in women: is the black box becoming grayer?
}

\author{
Wael Agur • Diaa E. E. Rizk
}

Received: 10 September 2010 /Accepted: 19 September 2010 /Published online: 6 October 2010

(C) The International Urogynecological Association 2010

"I have more flesh than another man, and therefore more frailty"

William Shakespeare (1564-1616).

The prevalence of obesity is increasing worldwide and has lately reached epidemic proportions in many countries. Epidemiological studies have consistently shown that both overweight and obesity are important risk factors for the development of various female pelvic floor disorders including urinary incontinence, pelvic organ prolapse, and fecal incontinence, as well as resistance to treatment [1]. The relationship between obesity and urinary incontinence will be discussed here.

Strong associations between obesity and stress urinary incontinence (SUI) in women had been frequently reported in the urogynecologic literature $[1,2]$. The pathophysiologic basis for this relationship is the significant correlation between body mass index (BMI) and intra-abdominal pressure suggesting that obesity may stress the pelvic floor secondary to a chronic state of increased pressure [2]. Obesity has also been confirmed as an important risk factor for failure of continence surgery in the first follow-up year. Patients with BMI $>30$ were 3.7 times more likely to be incontinent 1 year after colposuspension compared to nonobese patients [1]. Weight loss has been proven to be an effective treatment of SUI in obese women. Weight

\footnotetext{
W. Agur $(\bowtie)$

Department of Obstetrics and Gynecology, Crosshouse Hospital, Kilmarnock, Scotland, UK

e-mail: waelagur@nhs.net

D. E. E. Rizk

Department of Obstetrics and Gynecology, Faculty of Medicine, Ain Shams University,

Cairo, Egypt
}

reduction of only $5 \%$ of initial body weight has been shown to reduce UI severity enough to result in significant improvement in quality of life measures in obese women [3]. Moreover, surgically induced weight loss had been shown to significantly improve various urodynamic and bladder/urethral imaging parameters during coughing, e.g., the magnitude of vesical pressure increase, bladder-tourethra pressure transmission, and urethral axial mobility.

More recently, overweight and obesity were found to be an independent risk factor for overactive bladder $(\mathrm{OAB})$ in women [4]. The most likely explanation is the occurrence of chronic low-grade inflammation in the bladder of obese women indicated by increased urinary chemokines [5]. In fact, dietinduced obesity was shown to be associated with infiltration of macrophages into adipose tissue [6]. Adipocytes surrounding the human bladder can be affected, thus leading to inflammation and triggering $\mathrm{OAB}$ symptoms. The waist-hip ratio has been advocated as a measure of "central" obesity because BMI does not describe the distribution of obesity. This measurement, however, was found to be associated with SUI but not with $\mathrm{OAB}$ or mixed urinary incontinence, further suggesting a nonmechanical mechanism for OAB in obese women [7].

The association between urinary incontinence and diabetes mellitus, a disease that is closely linked to obesity, is well established $[1,2]$. It has been suggested that the most likely reason for the increased risk is a microvascular compromise in diabetic women leading to damage to the urethral sphincter mechanism and bladder "sensitivity" with increased risk of developing SUI and OAB, respectively. Stricter glycemic control may reduce the risk or severity of urinary incontinence [2]. Some studies have also found that women who used insulin were more likely to be incontinent than women who did not require insulin, but the mechanisms are unclear. 
Ghrelin is a recently discovered 28 amino acid peptide hormone that was initially identified as an endogenous ligand for growth hormone (GH) stimulating hormone receptor in rat and human stomach and is the only known orexigenic gut hormone $[8,9]$. Levels of circulating ghrelin have been noted to increase before meals and fall rapidly after eating [8]. Both central nervous system and peripheral administration of ghrelin increase food intake and body weight with a reduction in lipolysis in rodents. Fasting plasma levels of ghrelin are high in patients with anorexia nervosa and in subjects with diet-induced weight loss, whereas obese subjects demonstrate lower fasting ghrelin levels and postprandial ghrelin suppression. A decrease in circulating ghrelin has also been found following bypass operations and may be responsible for the long-term weight loss following bariatric surgery. Ghrelin receptors are expressed in the pituitary gland and hypothalamus as well as a wide variety of peripheral tissues including the urethra, urinary bladder, and anal canal of rats [8-10]. Recent animal studies have shown that ghrelin also has important anti-aging function on the pelvic floor [9]. Ghrelin administration reversed the expression of aging markers in the pelvic floor supportive structures of ovariectomized old rats either by acting through target organ ghrelin receptors or by stimulating GH release. Ghrelin also inhibited the in vitro contractile response of rat detrusor muscle, and the relaxant effect was more pronounced in strips obtained from lean rats than from obese rats [10]. It is therefore possible that the decrease in circulating ghrelin associated with obesity could adversely affect the urethral support and detrusor contractility in obese women to increase the risk of SUI and OAB, respectively $[9,10]$.

There are important clinical points to consider when managing obese women with urinary incontinence. Examination to demonstrate SUI and assess for associated pelvic organ prolapse could be difficult due to limited access or difficulties with positioning on unsuitable examination couches. Measuring post-void residual urine by a bladder scan usually requires two practitioners and may be less accurate in obese compared to non-obese women. Giving fluid advice is usually not easy as fluid matrices rarely provide guidance on the acceptable fluid intake for patients who weigh over $90 \mathrm{~kg}$. Higher doses of anti-muscarinic drugs may be required in the obese patient with $\mathrm{OAB}$ which in turn may increase the frequency of adverse effects. Although the minimally invasive surgical techniques (e.g., mid-urethral slings and urethral bulking agents for SUI and intra-vesical botulinum toxin injection for $\mathrm{OAB}$ ) have reduced surgical risks in obese patients, the anesthetic risks remain high and intermittent self-catheterization, if required, may be difficult. Attempting weight loss prior to surgical intervention is not always feasible in some obese patients for various practical reasons. In these cases, the operation can still be performed provided that the patient is fully aware of the higher risks involved and after taking necessary peri-operative precautions.

In conclusion, the global female obesity epidemic continues to grow, increasing the risks of pelvic floor dysfunction particularly urinary incontinence in women. In obese women, SUI may be secondary to mechanical pressure on the pelvic floor, whereas $\mathrm{OAB}$ appears to be associated with an inflammatory and chemical response in the urinary bladder. Coexistent diabetes mellitus is an additional risk factor for both types of urinary incontinence in obese women. The relationship between obesity and female urinary incontinence may also be related to reduction of circulating ghrelin levels with consequent adverse effects on urinary control. Although moderate weight loss results in improvement of quality of life and better outcome of surgical treatment in incontinent obese women, the management of these patients may be different than in non-obese women. Additional neurophysiologic and urodynamic studies are urgently needed to better define the mechanism of urinary incontinence in obese women.

\section{References}

1. Subak LL, Richter HE, Hunskaar S (2009) Obesity and urinary incontinence: epidemiology and clinical research update. J Urol 182(Suppl):S2-S7

2. Lawrence JM, Lukacz ES, Liu IA, Nager CW, Luner KM (2007) Pelvic floor disorders, diabetes, and obesity in women: findings from the Kaiser Permanente Continence Associated Risk Epidemiology Study. Diab Care 30:2536-2541

3. Auwad W, Steggles P, Bombieri L, Waterfield M, Wilkin T, Freeman R (2008) Moderate weight loss in obese women with urinary incontinence: a prospective longitudinal study. Int Urogynecol J 19:1251-1259

4. Hunskaar S (2008) A systematic review of overweight and obesity as risk factors and targets for clinical intervention for urinary incontinence in women. Neurourol Urodyn 27: 749-757

5. Tyagi P, Barclay D, Zamora R, Yoshimura N, Peters K, Vodovotz Y, et al. (2009) Urine cytokines suggest an inflammatory response in the overactive bladder: a pilot study. Int Urol Nephrol. doi:10.1007/s11255-009-9647-5

6. Neels JG, Olefsky JM (2006) Inflamed fat: what starts the fire? J Clin Invest 116:33-35

7. Townsend MK, Curhan GC, Resnick NM, Grodstein F (2008) BMI, waist circumference, and incident urinary incontinence in older women. Obesity 16:881-886

8. Suzuki K, Simpson KA, Minnion JS, Shillito JC, Bloom SR (2010) The role of gut hormones and the hypothalamus in appetite regulation. Endocr J 57:359-372

9. Rizk DEE, Fahim MA (2008) Ageing of the female pelvic floor: towards treatment "a la carte" of the "geripause". Int Urogynecol J 19:455-458

10. Chancellor MB, Oefelein MG, Vasvada S (2009) Obesity is associated with more severe overactive bladder disease state that is effectively treated with daily administration of trospium chloride extended release. Neurourol Urodyn 29:551-554 